\title{
Design of Programming Experiment Course Platform Based on MOOCs
}

https://doi.org/10.3991/ijet.v14i10.10330

\author{
Yu Zhao, Lifeng Guo, Hongxin Liu $\left({ }^{\varpi}\right)$ \\ Northeast Agricultural University, Harbin, China \\ Lcc98@ neau.edu.cn \\ Wenbin Zheng \\ Harbin Institute of Technology, Harbin, China
}

\begin{abstract}
It is the flourishing development of MOOCs that brings new mode of education. The paper focuses on the improvement of the teaching methods of programming experiment course in the environment of MOOCs, the development of experimental platform for innovative teaching methods, and the assessment methods of student learning. The current research and use of MOOCs are discussed, and its application in the programming experiment course. According to the present research analysis, the online programming experiment course platform is built, and a kind of online assessment system for students' achievement evaluation methods is elaborated. The academic performance and interest in learning are improved when experiment course platform is applied through two years teaching comparison. It is feasible to adopt the online platform for programming experiment course.
\end{abstract}

Keywords-Programming experiment course, online judge, MOOCs.

\section{Introduction}

The Massive Open Online Courses abbreviated MOOCs is open access via web and the participants are dispersed all over the world [1]. The Massachusetts Institute of Technology established the MIT Open Course Ware in 2002 and released the course to the Internet, then Stanford University and Harvard University also released online videos for free. MOOCs website emerged, such as Coursera, Udacity and edX, represented the arrival of large open online course in 2012, so 2012 was called "the year of the MOOC" according to the New York Times. Computer scientists at Stanford University founded Coursera which collaborates with world famous universities to promote students and attract students from all over the world. Coursera's courses were not only about online video transmission, but also required students to learn on schedule. Tests and discussion were interspersed during learning. edX was an online course system organized by Harvard University, MIT and the University of California at Berkeley [2]. edX announced a partnership with Google to develop MOOC.org, and Google will work with edX partners to develop core platforms in September 2013. 
The MOOCs has developed rapidly in recent years. The research on MOOC has the following aspects: the development history of MOOCs and its influence on traditional education; the research on the platform of MOOCs; the improvement of teaching method and teaching evaluation; quality assurance and credit certification of MOOCs; operation and management mode of MOOCs. Throughout MOOCs resources, computer-related courses occupy most, which show that the demand for computer courses is large and the acceptance of the course is better. At the same time, computer science is a series of courses that emphasizes practicality, especially the programming courses and their corresponding experimental courses. It is necessary to form the MOOCs courses of programming experiment courses.

The MOOCs bring a revolution in traditional education and a kind of new teaching model. Experimental teaching is an important method to cultivate students' innovative ability and master practical skills. Experimental teaching is restricted by many aspects such as venue, equipment and personnel, and it is not easy to realize sharing and open. With the development of the MOOCs, experimental teaching is also exploring new teaching models and methods. There are also many successful experiences in experimental teaching of open and sharing. For instance, computer-based hardware experiments can be opened and shared using virtual laboratory technology. Some universities have established virtual experimental resources through simulation software or virtual remote control experimental hardware, such as virtual engineering laboratory of Johns Hopkins University, the iLab online laboratory of Massachusetts Institute of Technology, etc. [3]. edX has a WebSim which is a simulation-based online electronic circuits laboratory offered by MIT, and learning assessment is evaluated through artificial intelligence software or student's mutual grade [4].

High-level language programming experiments such as $\mathrm{C}$, Java, and $\mathrm{C}++$, etc. do not need virtual instruments, while students can arrange time to upload code through the online platform to complete the experiment. However manual verification cannot meet the needs of large-scale experiments and the duplicate checking of experimental contents and the assessment of experimental results cannot be unified. The implementation of the programming experiment course based on MOOCs requires the corresponding technical platform and tools. The source codes of Open edX is open to the whole world, then people can deploy code to their own servers and build an online learning platform [5]. Dorsa designed automated problem generation, automated exercises and automated scoring for embedded system MOOC course[6]. De Waard analyzed the impact of mobile learning using communication terminals on user interaction in MOOCs [7]. In the programming competition, the online judge system evaluates the code from the correctness, time consuming, memory cost and return results of the program [8]. In addition, some universities use the online judge system of programming competition and adopt distributed system architecture and corresponding optimized strategies to design teaching experiment platform.

Although there are many advantages in the open sharing of the MOOCs, some problems have also been discovered in the discussion with the traditional teaching: the MOOCs lack the supervision mechanism of the students, many students give up the course; the online course can provide excellent teaching resources, however there is a lack of communication between students and teachers; the MOOCs face large-scale 
student groups and it is difficult for teachers to take care of individuals; it is hard to provide high-level innovative practical experiences for students[9]. The article combines the advantages of the MOOCs with the local traditional higher education, promoting each other. Advanced language programming experimental learning and theoretical learning are complementary according to computer programming course. The paper develops a system of integrated online courses and online assessment, integrating present high-quality experimental courses and a unified experimental platform to achieve experimental courses open and shared.

\section{Online Course Learning Platform}

High-quality curriculum resources were established and representative teaching videos were selected. The learning process of the online course learning platform can be described as follows: the students need to learn the theoretical knowledge and experimental content needed for the experiment in advance, and for each experimental content the students submit the code through the experimental platform. The online assessment system evaluates the code. The learning effect is obtained through the questions answered by students, the quality of the experimental code, the test results, and the interaction of the community. The design of the experimental platform should realize experiment sharing, specification management, progress tracking, online assessment, interactive communication, courseware teaching and effect evaluation. The system structure diagram is shown in the Figure 1.

\subsection{Web and mobile communication terminal interface}

In order to access to the integrated experimental platform, web access supports windows, linux and mac operating system. Mobile terminal supports and smart phone with Android or iOS.

\subsection{User service management}

The platform adopts three levels of user management. Assign different access rights to different users. The system administrator has the highest authority, the teacher has second and the student has the lowest authority.

\subsection{Course management}

Students can browse and retrieve the experimental lessons provided by the platform in real time. Teachers and administrators select high-quality courses from the open resources to upload to the platform, and teachers can post the recorded instructional videos to the experimental platform. 


\subsection{Interactive teaching management}

Through interactive teaching management, students start online experimental courses, the effective learning time and progress of the students are confirmed through technical methods. The teachers insert the questions prepared in advance into the course, then the students answer in real time. The teachers can record the teaching process to make new videos and courseware posted on the platform.

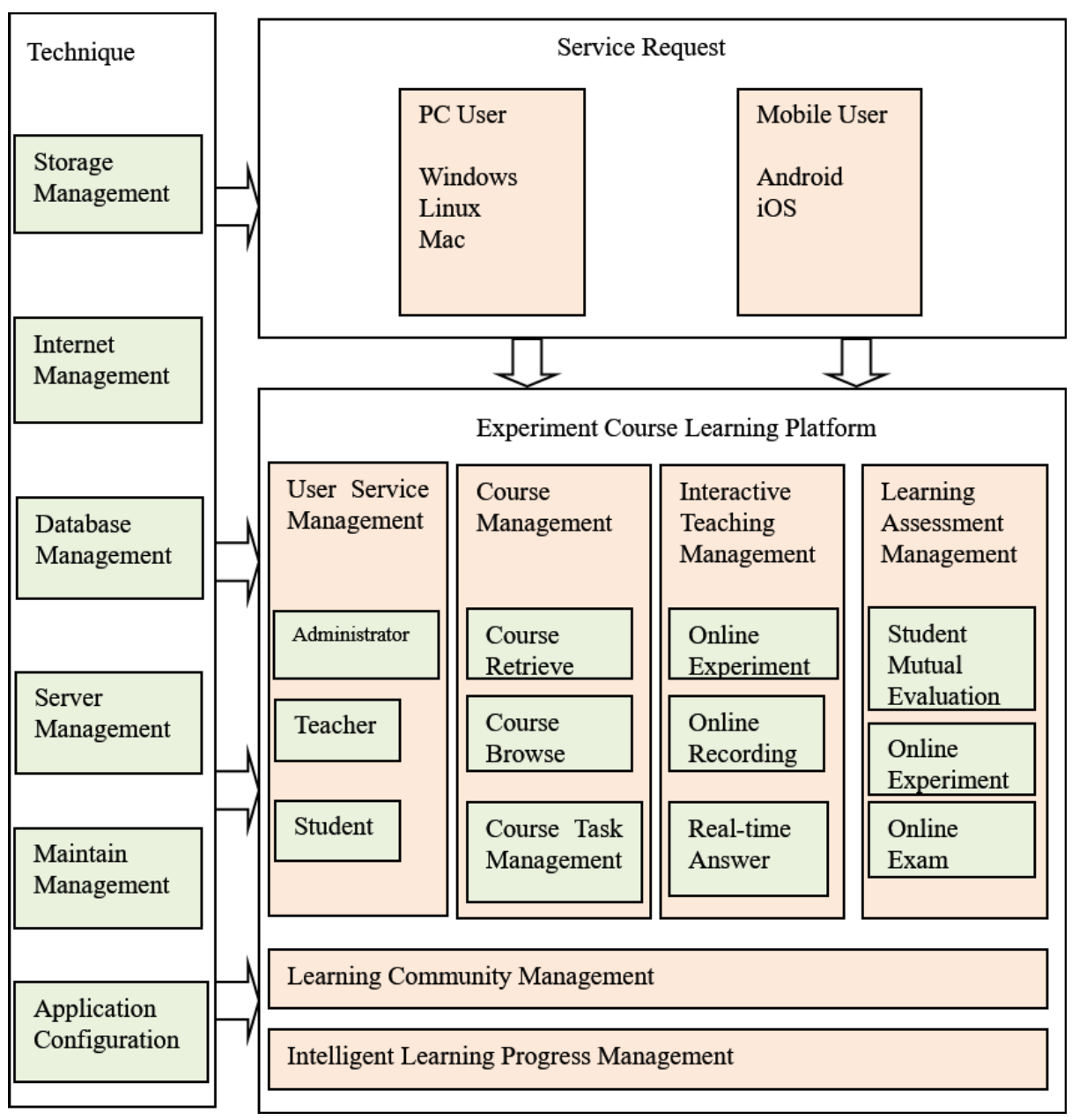

Fig. 1. The system structure diagram

\subsection{Learning assessment management}

For each experimental content, online judge system is designed to give the evaluation results. For the group experiments that need to be completed by cooperation, mutual scoring is adopted to effectively improve the learning efficiency. Each group not 
only masters the algorithm design but also is familiar with the algorithm design of other groups. At the end of the semester, the course score is given by the online test system of real-time evaluation program. The assessment of learning effects consists of online learning time, interactive group evaluation, online experiment assessment and online examination.

\subsection{Intelligent learning progress management}

The learning unit designed according to the syllabus is arranged to release the course content and schedule to the students. Data analysis of the questions and learning content will be used to judge the strong and weak points of students' learning. For those who are good at it, a higher level of learning contents is recommend, so that it can be more deeply understood and applied. For those who are weak at it, they will also be guided by the specificity to make a balanced development.

\subsection{Learning community management}

The learning community provides a platform for teachers and students to communicate. Users with authority can establish or participate in the interested communities and can discuss freely. Each community has administrators who are responsible for the management and construction of the community. The community also has reward points management for quality resources, and can ask and answer questions online.

\section{Course Online Judge System}

Traditional experimental methods are hard to check and answer students' questions one by one. In order to improve the teaching quality of experimental teaching, automatic evaluation and automated inspection methods are introduced in the learning assessment management. The online judge system belongs to the learning assessment management in Figure 1. The management and scheduling resources are developed with relatively well-developed Python language, and the performance-critical part of the system are developed with the more efficient $C$ language in the system design. User obtains the questions through the browser and the code submitted after the completion of the question is directly saved in the database.

The online judge system is not only used to check the accuracy of the program code written by the students in each lab session but also to check the code accuracy of the final exam. It is divided into user management module, question setting module, judge module, answer module, score management module and log management module. The system class diagram is shown in Figure 2.

The user management module verifies if the user identity is legal and has the appropriate permissions.

The teachers classify and publish the questions in question setting module, each question has corresponding test data which is invisible to the students. 
The judge module evaluates the results of the questions based on the test data and the evaluation strategy.

According to the classification of the questions, the answer module selects the questions with same level from the question database randomly and assigns them to students, which reduces the probability that different students get the same questions and prevents code plagiarism.

The score management module provides results query and print function.

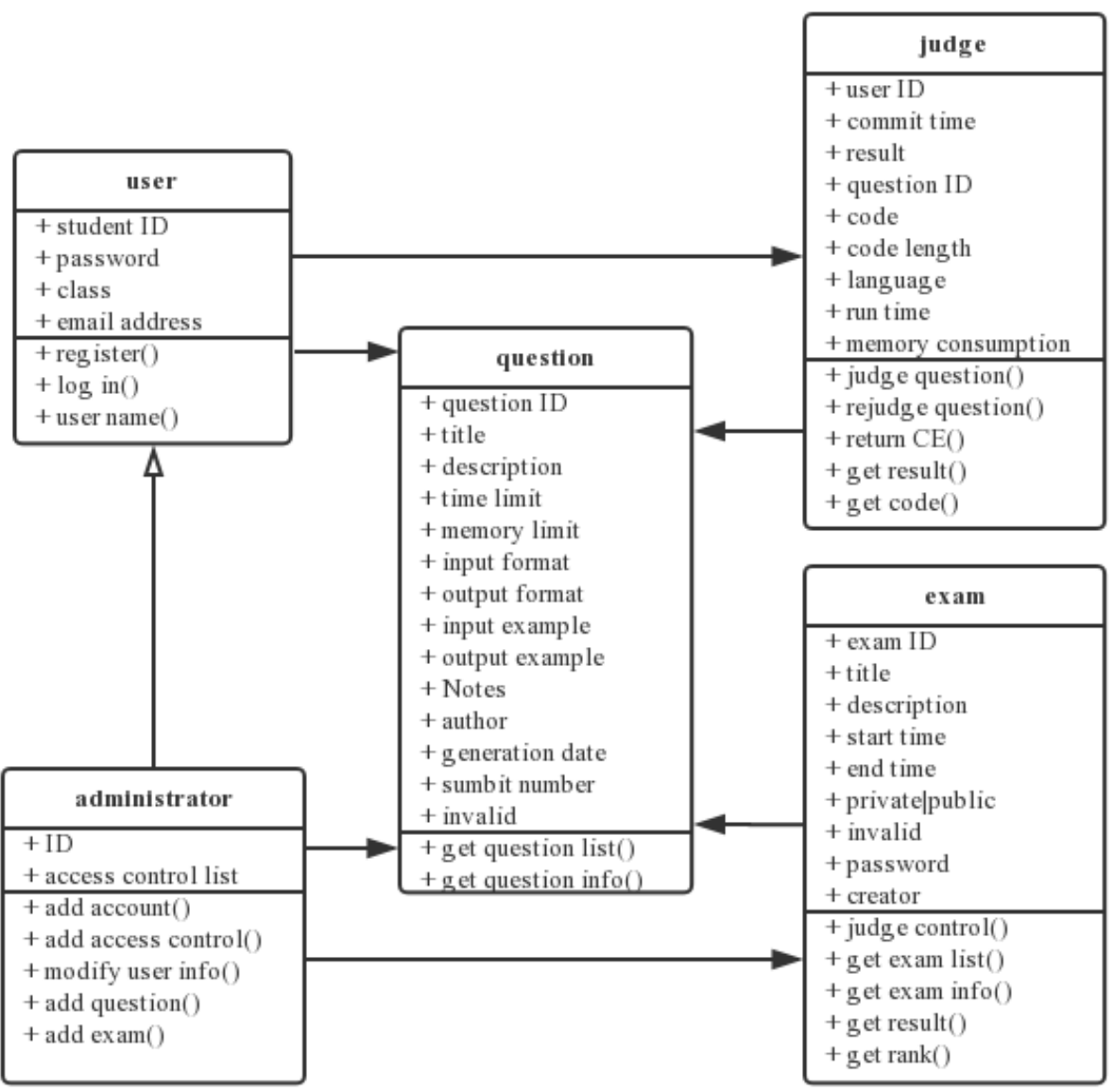

Fig. 2. Online judge class diagram

The judging strategy is to perform a black-box test on the submitted program to generate a judgment result. The system compiles and runs the source code, and verifies the correctness of the code with test data. As the same time, the system monitors the amount of memory and runtime while the program is running.

The judging kernel uses the producer-consumer model to prevent deadlocks caused by multiple judging threads judge the same program simultaneously, while a thread queue maintains the judging thread. When the daemon gets the signal sent by the web 
the main process generates a producer thread and puts it into the task queue. If the task queue is full the producer thread will be blocked until a consumer thread completes the judging job and releases the resource. There are multiple judging threads in the consumer thread pool. When there is a production request in the task queue the consumer will get the task from the task queue. Once a thread gets a task from the queue other threads cannot get the task again. If the task queue is empty, the task cannot be obtained. At this time the judging thread is blocked waiting for the arrival of the task. A blocked thread can be thought of as a stoppage which can significantly reduce the consumption of system resources.

When a judging thread gets the task the thread will be woken up and the code will be judged. First, the judge module takes the code corresponding to the task from the database and saves the file to the corresponding judge directory according to the code language. Then it compiles the code in the judge directory. If the code is compiled incorrectly, the error information is saved in the database and returned error. If the compiler passes, the program will be run. In order to prevent malicious code from being executed, the sandbox is set up. When the program is running, the execution time and memory of the program are monitored.

\section{$4 \quad$ Experiment and Results}

In order to verify the effectiveness of the platform, the C language programming experiment course was designed. After the software platform was developed, the $\mathrm{C}$ language programming experiment teaching and testing resources were designed and the administrator released the prepared course resources to the platform.

Course management is divided into course navigation, course editing, and course task management. In the course navigation module students enroll in courses, download courseware, submit course, assignments and submit group evaluations.

In the course editing module, teachers and administrators set teaching content and course information, manage course resources such as video production, video distribution, multimedia resource decoding and compression.

In the course task management module, the teacher manages the elective course student information and formulates the group information. After class there is a learning community for students to discuss and communicate. The community management module is divided into user management module, system maintenance module, user information module, collaborative communication module, information module and $\log$ management module. At the end of the semester the assessment of learning effectiveness consists of online learning time, interactive group evaluation, online experimental judging and online examination.

The results of the after-class survey show that the teaching method can improve the initiative and enthusiasm of students' learning and improve the interest of learning. The students have gained more knowledge and achieved good results. 


\section{Conclusion}

The paper develops a set of experimental teaching platform for integrated teaching and assessment based on the actual course situation which will effectively lay foundation for the promotion of experimental MOOCs development.

A teaching method and teaching assessment method are proposed for computer programming experimental courses based on MOOCs. Students use the experimental platform built on campus to learn independently and complete the relevant experimental content and release code. Teaching assessment strategy of this method is discussed and the platform gives the assessment automatically. The platform embodies the purpose and significance of online courses and integrates teaching and learning which is more conducive to the development of online education.

The online judge system is integrated into the experimental course platform so that it can realize teaching, learning and assessment automatically. The programming course emphasizes the programming ability and it is impossible for the teacher to check the code of all the students line by line. The online judge system automatically evaluates the code in the black box test mode and promotes it to the programming platform of the programming class.

\section{Acknowledgement}

This work is supported in part by the Heilongjiang Postdoctoral Fund (Grant No. LBH-Z16081) and Higher Education Scientific Research Topic of Heilongjiang Higher Education Society in the 13th Five-Year Plan (Grant No. 16Q004).

\section{$7 \quad$ References}

[1] Kaplan AM, Haenlein M. Higher education and the digital revolution: About MOOCs, SPOCs, social media, and the Cookie Monster. Business Horizons. 2016; 59:441-50. https://doi.org/10.1016/j.bushor.2016.03.008

[2] Breslow L, Pritchard DE, Deboer J, Stump GS, Ho AD, Seaton DT. Studying Learning in the Worldwide Classroom Research into edX's First MOOC. Research \& Practice in Assessment. 2013;8:13-25.

[3] A VIRTUAL ENGINEERING/SCIENCE LABORATORY COURSE http://wwwjhuedu/virtlab/virtlabhtml

[4] WebSim. http://euryalecsailmitedu/.

[5] Grover S, Pea R, Cooper S. Promoting active learning \& leveraging dashboards for curriculum assessment in an OpenEdX introductory CS course for middle school. ACM Conference on Learning @ Scale Conference2014. p. 205-6. https://doi.org/10.1 $\underline{145 / 2556325.2567883}$

[6] Sadigh D, Seshia SA, Gupta M. Automating exercise generation: A step towards meeting the MOOC challenge for embedded systems. The Workshop on Embedded \& Cyber-Physical Systems Education2012. p. 1-8. https://doi.org/10.1145/2530544.2530546

[7] De Waard I. Analyzing the impact of mobile access on learner interactions in a MOOC: Athabasca University; 2013. 
[8] Peking University Judge Online For ACIP/ICPC. http://pojorg/.

[9] Rizvi S. An avalanche is coming: Higher education and the revolution ahead. Technology. 2012.

\section{Authors}

Yu Zhao is with School of Electrical and Information, Northeast Agricultural University, No.600 Changjiang Street, Harbin, Heilongjiang Province, China, 150030. (Email: yuzhao@neau.edu.cn).

Lifeng Guo is with School of Engineering, Northeast Agricultural University, No.600 Changjiang Street, Harbin, Heilongjiang Province, China, 150030.

Wenbin Zheng is with School of Electronics and Information Engineering, Harbin Institute of Technology, No.2 Yikuang Street, Harbin, Heilongjiang Province, China, 150080.

Hongxin Liu is with School of Engineering, Northeast Agricultural University, No.600 Changjiang Street, Harbin, Heilongjiang Province, China, 150030.

Article submitted 2019-02-15. Resubmitted 2019-03-26. Final acceptance 2019-04-01. Final version published as submitted by the authors. 